\title{
METHOD OF EXTREMAL SHIFT \\ IN PROBLEMS OF RECONSTRUCTION \\ OF AN INPUT FOR PARABOLIC VARIATIONAL INEQUALITIES
}

\author{
Vyacheslav Maksimov* \\ Institute of Mathematics and Mechanics, \\ Ural Branch, Russian Academy of Sciences, \\ S. Kovalevskoi str., 16, Ekaterinburg, 620219 Russia \\ maksimov@imm.uran.ru
}

\begin{abstract}
The problem of dynamical reconstruction of a control in a parabolic variational inequality [1-4] through inaccurate measurement of phase state is considered. This problem belongs to the class of dynamical inverse problems which consist in reconstruction of unknown input (a control) of dynamical systems from (inaccurate) measurement of outputs.
\end{abstract}

Keywords: reconstruction, parabolic inequality

\section{Introduction}

Let $V$ and $H$ be real Hilbert spaces, $V$ be a dense subspace of $H$ and $V \subset H=H^{*} \subset V^{*}$ algebraically and topologically. We denote by $|\cdot|_{V}$ and $|\cdot|_{H}$ the norms in $V$ and $H$, respectively, by $(\cdot, \cdot)$ a scalar product in $H$, and by $\langle\cdot, \cdot\rangle$ duality between $V$ and its dual $V^{*}$. Let a dynamical system be described by the parabolic variational inequality

$$
\begin{gathered}
\left(x_{t}(t)-f(t), x(t)-z\right)+\langle A x(t)-B u(t), x(t)-z\rangle+\varphi(x(t))-\varphi(z) \leq 0 \\
\text { a. e. } t \in T=\left[t_{0}, \vartheta\right] \forall z \in V, x\left(t_{0}\right)=x_{0} \in D(\varphi) .
\end{gathered}
$$

Here, $A: V \rightarrow V^{*}$ is a linear continuous symmetrical operator satisfying (with a certain $c>0$ and $\omega \in R$ ) the following condition

$$
\langle A y, y\rangle+\omega|y|_{H}^{2} \geq c|y|_{V}^{2}
$$

${ }^{*}$ This work was supported in part by the Russian Foundation for Basic Research (grant \# 01-01-00566).

The original version of this chapter was revised: The copyright line was incorrect. This has been corrected. The Erratum to this chapter is available at DOI: 10.1007/978-0-387-35690-7_44 
$f(\cdot) \in W^{1,2}(T ; H)$ is a given disturbance; $\varphi: V \rightarrow R^{+}=\{r \in R: r \geq 0\}$ $\cup\{+\infty\}$ is a weakly lower semicontinuous convex function, $D(\varphi)=$ $\{x \in V: \varphi(x)<+\infty\}, U$ is a uniformly convex Banach space (the space of controls), $W^{1,2}(T ; H)=\left\{x(\cdot) \in L_{2}(T ; H): x_{t}(\cdot) \in L_{2}(T ; H)\right\}$, the derivative $x_{t}(\cdot)$ is understood in a sense of distribution; $B$ is a linear continuous operator from $U$ to $H$.

Introduce a function $\varphi_{\omega}(y): H \rightarrow R^{+}$,

$$
\varphi_{\omega}(y)= \begin{cases}1 / 2\langle A y, y\rangle+\omega / 2|y|_{H}^{2}+\varphi(y), & \text { if } y \in D(\varphi) \\ +\infty & \text { otherwise. }\end{cases}
$$

From Theorem 4.1, 1.13 [2] it follows that for any $u(\cdot) \in L_{2}(T, U)$, $x_{0} \in D(\varphi)$ there exists a unique solution of the inequality (1) with the properties: $x(\cdot)=x\left(\cdot ; t_{0}, x_{0}, u(\cdot)\right) \in W_{*}(T)=W^{1,2}(T ; H) \cap L_{2}(T ; V)$, $x(t) \in D\left(\varphi_{\omega}\right) \quad \forall t \in T, \quad t \rightarrow \varphi_{\omega}(x(t)) \in A C(T)$. Here $A C(T)$ is a space of absolutely continous functions.

The problem may be formulated in the following way. Let a uniform net $\Delta=\left\{\tau_{i}\right\}_{i=0}^{m}, \tau_{i}=\tau_{i-1}+\delta, \tau_{0}=t_{0}, \tau_{m}=\vartheta$ be fixed on a given time interval $T$. Let a motion of system (1) proceed on the interval $T$. Its trajectory (the solution of $(1)) x_{r}(\cdot)=x\left(\cdot ; t_{0}, x_{0}, u_{r}(\cdot)\right)$ depends on time-varying unknown control $u(\cdot)=u_{r}(\cdot) \in U_{T}=L_{2}(T ; U)$. Here $U_{T}$ is a set of admissible controls. The trajectory $x_{r}(\cdot)$ is unknown. At moments $\tau_{i}$ the phase state $x_{r}\left(\tau_{i}\right)$ is inaccurately measured. The results of measurements $\xi_{i}^{h} \in H, i \in[0: m-1]$ satisfy the inequality

$$
\left|\xi_{i}^{h}-x_{r}\left(\tau_{i}\right)\right|_{H} \leq h \text {. }
$$

Here, $h \in(0,1)$ is the value of the level of informational noise. It is required to design an algorithm allowing to reconstruct (synchro with the process) some unknown input $u_{*}(\cdot)$ generating solution $x_{r}(\cdot)$ to inequality (1). This is meaningful statement of the problem.

\section{The approach to solving the problem}

One of the approaches to solving the problems of similar type was described, for example, in [5-12]. (See more detail on this approach in surweys [13-16]). Briefly remind about the essence of this approach. Let $U\left(x_{r}(\cdot)\right)$ be the set of all inputs $u(\cdot) \in U_{T}$ which are compatible with $x_{r}(\cdot), \Xi_{T}$ be the set of all measurements, i. e. the set of all piece-wise functions on $T$ with values in $H, \Xi\left(x_{r}(\cdot), h\right)$ be the set of all $h$-accurate measurements, i. e. functions $\xi^{h}(\cdot) \in \Xi_{T}$ satisfying (3). An auxiliary system $M$ (a model) described by a parabolic variational inequality of the form

$$
\begin{gathered}
\left(w_{t}^{h}(t)-f(t), w^{h}(t)-z\right)+\left\langle A w(t)-B_{t}\left(w^{h}(\cdot), \xi^{h}(\cdot)\right) v^{h}(t), w^{h}(t)-z\right\rangle+ \\
\varphi\left(w^{h}(t)\right)-\varphi(z) \leq 0 \text { a. e. } t \in T \forall z \in V, w^{h}\left(t_{0}\right)=w_{0}^{h} \in D(\varphi)
\end{gathered}
$$


is considered. Here $B_{t}(\cdot, \cdot): U \rightarrow H$ is a family of operators, whose structure will be specify below. The motion of the model is denoted as $w^{h}(\cdot)=w^{h}\left(\cdot ; t_{0}, w_{0}^{h}, v^{h}(\cdot)\right) \in W_{*}(T)$. Here $v^{h}(\cdot)$ is a control in the model. Initial state $w_{0}^{h}$ of the model is chosen by using the value $\xi_{0}^{h}$ of measurement at initial time moment $t_{0}$ in accordance with some rule $\mathcal{W}_{h}$ fixed in advance:

$$
w_{0}^{h}=w^{h}\left(t_{0}\right)=\mathcal{W}_{h}\left(\xi_{0}^{h}\right) \in H .
$$

Model control rules are identified with pairs $S_{h}=\left(\Delta_{h}, \mathcal{U}_{h}\right)$, where $\Delta_{h}=$ $\left\{\tau_{h, i}\right\}_{i=0}^{m_{h}}$ is a partition of the interval $T$ into half-intervals $\left[\tau_{h, i}, \tau_{h, i+1}\right)$, $\tau_{h, i+1}=\tau_{h, i}+\delta, \delta=\delta(h), \tau_{h, 0}=t_{0}, \tau_{h, m_{h}}=\vartheta, \mathcal{U}_{h}$ is a function relating element

$$
v_{i}^{h}=\mathcal{U}_{h}\left(\tau_{i}, \xi_{i}^{h}, w^{h}\left(\tau_{i}\right)\right) \in U
$$

to every triple $\left(\tau_{i}, \xi_{i}^{h}, w^{h}\left(\tau_{i}\right)\right), i \in\left[0: m_{h}-1\right]$, where $\tau_{i}=\tau_{h, i}, w^{h}\left(\tau_{i}\right)=$ $w^{h}\left(\tau_{i} ; t_{0}, w_{0}^{h}, v^{h}(\cdot)\right), \xi_{i}^{h}=\xi^{h}\left(\tau_{i}\right), \xi^{h}(\cdot) \in \Xi\left(x_{r}(\cdot), h\right)$. Thus, quadruple $\left(M, \mathcal{W}_{h}, \Delta_{h}, \mathcal{U}_{h}\right)$ for every $h \in(0,1)$ determines some algorithm $D_{h}$ on space of measurements $\xi(\cdot) \in \Xi\left(x_{r}(\cdot), h\right)\left(D_{h}: \Xi_{T} \rightarrow U_{T}\right)$ forming output $v^{h}(\cdot)=D_{h} \xi(\cdot)$ according to the feedback principle (4)-(6). We identify the algorithm $D_{h}$ with quadruple $\left(M, \mathcal{W}_{h}, \Delta_{h}, \mathcal{U}_{h}\right)$. The result of work of the algorithm on the interval $T$ is a piecewise constant control $v^{h}(\cdot)$ of the form

$$
v^{h}(t)=v_{i}^{h}, \quad t \in\left[\tau_{i}, \tau_{i+1}\right) .
$$

Let the following condition be fulfilled.

Condition 1 The set $U_{*}\left(x_{r}(\cdot)\right)$ of $L_{2}(T ; U)$-norm minimal inputs in $U\left(x_{r}(\cdot)\right)$ is one-element, i. e. $U_{*}\left(x_{r}(\cdot)\right)=\left\{u_{*}\left(\cdot ; x_{r}(\cdot)\right)\right\}$.

A family $D_{h}, h \in(0,1)$ of operators from $\Xi_{T}$ to $U_{T}$ is called regularizing if

$$
\lim _{h \rightarrow 0} \sup \left\{\left|D_{h} \xi^{h}(\cdot)-u_{*}\left(\cdot ; x_{r}(\cdot)\right)\right|_{L_{2}(T ; U)}: \xi^{h}(\cdot) \in \Xi\left(x_{r}(\cdot), h\right)\right\}=0 .
$$

We consider the problems of construction of regularizing families of algorithms of modeling

$$
D_{h}=\left(M, \mathcal{W}_{h}, \Delta_{h}, \mathcal{U}_{h}\right), \quad h \in(0,1)
$$

of the form (4)-(7). We call them positional algorithms of modeling.

For parabolic variational inequality in [9-11], some regularazing families of algorithms of modeling $D_{h}$ of type (8) were indicated for the case when the set of admissible controls $U_{T}$ has the form: $U_{T}=\{u(\cdot) \in$ $L_{2}(T ; U): u(t) \in P$ for a.e. $\left.t \in T\right\}$. Here $P \subset U$ is a convex, bounded, and closed set. The constructions of works [9-11] are based on combination of the theory of control with a model [17] and the known in 
the theory of ill-posed problems method of smoothing functional (also often called Tikhonov's method). In the present work, we modify algorithms $[5-8,10-12]$ for the case when set $U_{T}$ is unbounded. Note that works $[9,13]$ indicate some other regularizing algorithms $D_{h}$ of reconstruction of unbounded controls in parabolic variational inequalities based on a dynamical modification of the discrepancy method. Questions on program control of parabolic variational inequalities have been discussed in monographs [2].

After model (4) and its initial state (5) are chosen the work of the algorithm $D_{h}$ (for fixed $h$ ) corresponds to the following outline. First, before the moment $t_{0}$, a partition $\Delta=\Delta_{h}=\left\{\tau_{i}\right\}_{i=0}^{m},\left(\tau_{i}=\tau_{h, i}\right)$ of the interval $T$ is chosen and fixed. At the $i$-th step carried out during the time interval $\left[\tau_{i}, \tau_{i+1}\right)$, the following sequence of actions takes place. The output $x_{r}\left(\tau_{i}\right)$ is inaccurately measured, i. e. the value $\xi_{i}^{h} \in H$ with the properties (3) is calculated. Then the model control is determined by (6), (7) and after that we form the new part of the model trajectory $w^{h}(t)$, $t \in\left(\tau_{i}, \tau_{i+1}\right]$ instead of $w_{t_{0}, \tau_{i}}^{h}(\cdot)$ (memory correction). The procedure stops at the time moment $\vartheta$.

Construction of a family $D_{h}$ is based on Theorem 1 formulated below. Let us fix a functional $\Lambda^{0}(\cdot, \cdot)$ on $W_{*}(T) \times W_{*}(T)$.

Definition 1 [7, 15] A family $D_{h}, h \in(0,1)$, (8) of positional algorithms of modeling is said to be $\Lambda^{0}$-stable if there exist functions $k_{1}(\cdot), k_{2}(\cdot)$, $k_{3}(\cdot):[0, \infty) \rightarrow[0, \infty)$ such that $k_{1}(h) \rightarrow 1, k_{2}(h) \rightarrow 0, k_{3}(h) \rightarrow 0$ as $h \rightarrow 0$, and for every observation record $\xi^{h}(\cdot) \in \Xi\left(x_{r}(\cdot), h\right)$, it holds that

$$
\begin{gathered}
\left|v^{h}(\cdot)\right|_{L_{2}(T ; U)} \leq k_{1}(h)\left|u_{*}\left(\cdot ; x_{r}(\cdot)\right)\right|_{L_{2}(T ; U)}+k_{2}(h), \\
\Lambda^{0}\left(x_{r}(\cdot), w^{h}(\cdot)\right) \leq k_{3}(h),
\end{gathered}
$$

where $v^{h}(\cdot)=D_{h} \xi^{h}(\cdot)$ and $w^{h}(\cdot)$ is the model motion generated by $D_{h}$ under the observation record $\xi^{h}(\cdot)$.

Then the following theorem is true.

Theorem 1 [15] Let a family $D_{h}$ of positional algorithms of modeling be a) $\Lambda^{0}$-stable, and b) for every $h_{k}>0, \quad h_{k} \rightarrow 0+$ as $k \rightarrow+\infty, \xi^{h_{k}}(\cdot) \in$ $\Xi\left(x_{r}(\cdot), h_{k}\right), w^{h_{k}}(\cdot)=w^{h_{k}}\left(\cdot ; t_{0}, w^{h_{k}}\left(t_{0}\right), v^{h_{k}}(\cdot)\right), v^{h_{k}}(\cdot)=D_{h_{k}} \xi^{h_{k}}(\cdot)$, the limit correlations $v^{h_{k}}(\cdot) \rightarrow v(\cdot)$ weakly in $L_{2}(T ; U), \Lambda^{0}\left(x_{r}(\cdot), w^{h_{k}}(\cdot)\right) \rightarrow 0$ imply that $v(\cdot) \in U\left(x_{r}(\cdot)\right)$. Then the family $D_{h}, h \in(0,1)$, is regularizing.

\section{Case $u_{*}\left(\cdot ; x_{r}(\cdot)\right) \in L_{\infty}(T ; U)$}

First we consider a case where a control $u_{*}\left(\cdot ; x_{r}(\cdot)\right)$ is a bounded function, i.e., $u_{*}\left(\cdot ; x_{r}(\cdot)\right) \in L_{\infty}(T ; U)$. Let a number $a>0$ such that $x_{0} \in X_{0}=\left\{x \in D(\varphi):|x|_{H}^{2}+\varphi_{\omega}(x) \leq a<+\infty\right\}$ be choosen. 
We take a family of partitions $\left(\Delta_{h}\right)$ of the interval $T$, and fuctions $\alpha(h): R^{+} \rightarrow R^{+}$and $d(h): R^{+} \rightarrow R^{+}$satisfying the following conditions:

$$
\begin{gathered}
\alpha(h) \rightarrow 0, \quad \delta(h) \rightarrow 0, \quad d(h) \rightarrow+\infty, \\
d(h)(h+\delta(h)) / \alpha(h) \rightarrow 0, \quad \alpha(h) d^{2}(h) \rightarrow 0 \text { as } h \rightarrow 0+.
\end{gathered}
$$

This condition holds, for example, if $\delta(h) \leq c h, \alpha(h)=c_{1} h^{\gamma}$, $\gamma=0,5+\gamma_{0}, \gamma_{0} \in(0 ; 0,25), d(h)=c_{2} h^{-1 / 4}, c, c_{1}, c_{2}$ are positive constants. The family $\left(\mathcal{W}_{h}\right)$ of $t_{0}$-algorithms is defined by rule $(5)$, where

$$
w_{0}^{h} \in B\left(\xi_{0}^{h}\right)=\left\{x \in X_{0}:\left|\xi_{0}^{h}-x\right|_{H} \leq 2 h\right\} .
$$

From (3) and inclusion $x_{0} \in X_{0}$, it follows $B\left(\xi_{0}^{h}\right) \neq \emptyset$. In its turn, the model control law $S_{h}=\left(\Delta_{h}, \mathcal{U}_{h}\right)$ is defined following rule (6), (7), where we assume

$$
\begin{gathered}
\mathcal{U}_{h}\left(\tau_{i}, \xi_{i}^{h}, w^{h}\left(\tau_{i}\right)\right)=\arg \min \left\{l\left(\alpha, v, s_{i}\right): v \in S(d(h))\right\} \\
l\left(\alpha, v, s_{i}\right)=2\left(s_{i}, B v\right)+\alpha(h)|v|_{U}^{2}, \quad s_{i}=w^{h}\left(\tau_{i}\right)-\xi_{i}^{h} \\
S(d(h))=\left\{u \in U:|u|_{U} \leq d(h)\right\} .
\end{gathered}
$$

For a fixed $h$ (and, consequently, a fixed family $\Delta_{h}=\left\{\tau_{i}\right\}_{i=0}^{m_{h}}$ with diameter $\delta(h)$ ) model $M$ is given by the mapping associated with each triple $\left(w_{0}^{h}, \xi^{h}(\cdot), v^{h}(\cdot)\right), w_{0}^{h} \in X_{0}, \xi^{h}(\cdot) \in \Xi(x(\cdot), h), v^{h}(\cdot) \in L_{2}(T ; U)$ with a function $w^{h}(\cdot)=w^{h}\left(\cdot ; t_{0}, w_{0}^{h}, \xi^{h}(\cdot), v^{h}(\cdot)\right) \in W_{*}(T)$ which is a unique solution of variational inequality (4), where

$$
B_{t}\left(\xi^{h}(\cdot), w^{h}(\cdot)\right) v^{h}(t)=B v^{h}(t)-\omega^{*}\left(w^{h}\left(\tau_{i}\right)-\xi_{i}^{h}\right)
$$

for $t \in \delta_{i}=\left[\tau_{i}, \tau_{i+1}\right), \tau_{i}=\tau_{h, i}, \xi_{i}^{h}=\xi^{h}\left(\tau_{i}\right), \omega^{*}=0$, if $\omega \leq 0, \omega^{*}=\omega+\varepsilon_{0}$, otherwise $\left(\varepsilon_{0} \geq 0\right)$. Let

$$
\Lambda^{0}\left(x(\cdot), w^{h}(\cdot)\right)=\left|x(\cdot)-w^{h}(\cdot)\right|_{C(T ; H)}^{2}+2 c\left|x(\cdot)-w^{h}(\cdot)\right|_{L_{2}(T ; V)}^{2} .
$$

Theorem 2 The family of positional modeling algorithms $D_{h}(8)$ of the form (4)-(7), (11)-(14) satisfies conditions of Theorem 1 and is regularizing.

Before proving the theorem, we formulate auxiliary statements. Let $\omega_{*}=\omega$, if $\omega>0, \omega_{*}=0$ otherwise. Introduce a mapping $l(\cdot): W_{*}(T) \rightarrow R^{+}$,

$$
l(y(\cdot))=|y(\cdot)|_{C(T ; H)}+\left|y_{t}(\cdot)\right|_{L_{2}(T ; H)}+|y(\cdot)|_{L_{2}(T ; V)} .
$$

The following lemmas are true.

Lemma 1 There exists a number $K_{*}=K_{*}\left(\omega_{*}, c,|B|_{\mathcal{L}(T ; U)}\right)$ such that for any $x_{0} \in D(\varphi), u(\cdot) \in U_{T}, x(\cdot)=x\left(\cdot ; t_{0}, x_{0}, u(\cdot)\right)$ the inequality 
holds.

$$
l(x(\cdot)) \leq K_{*}\left(1+\omega_{*}\left|x_{0}\right|_{H}+\varphi_{\omega}^{1 / 2}\left(x_{0}\right)+|u(\cdot)|_{L_{2}(T ; U)}\right)
$$

Lemma 2 There exists a number $K^{*}=K^{*}\left(\omega_{*}, c,|B|_{\mathcal{L}(T ; U)}, \varepsilon_{0}\right)$ such that for any $w_{0}^{h} \in D(\varphi), v^{h}(\cdot) \in U_{T}, \xi^{h}(\cdot) \in \Xi\left(x_{r}(\cdot), h\right), w^{h}(\cdot)=$ $w^{h}\left(\cdot ; t_{0}, w_{0}^{h}, \xi^{h}(\cdot), v^{h}(\cdot)\right)$, the inequality

holds.

$$
l\left(w^{h}(\cdot)\right) \leq K^{*}\left(1+\omega_{*}\left|w_{0}^{h}\right|_{H}+\varphi_{\omega}^{1 / 2}\left(w_{0}^{h}\right)+\left|v^{h}(\cdot)\right|_{L_{2}(T ; U)}\right)
$$

These lemmas are proved by the standard scheme $[1,2]$.

Let us emphasize that constants $K_{*}$ and $K^{*}$ do not depend on $w_{0}^{h}$, $\xi^{h}(\cdot), u(\cdot)$ or $v^{h}(\cdot)$.

Proof of Theorem 2. Let us show that the family $D_{h}(4)-(7),(11)-$ (14) is $\Lambda^{0}$-stable. Let $\xi^{h}(\cdot) \in \Xi\left(x_{r}(\cdot), h\right), v^{h}(\cdot)=D_{h} \xi^{h}(\cdot)$ and $w^{h}(\cdot)$ is the model motion (4) generated by $D_{h}$ under the observation record $\xi^{h}(\cdot)$. Consider the value $\varepsilon_{h}(t)=\left|\mu^{h}(t)\right|_{H}^{2}+2 c\left|\mu^{h}(\cdot)\right|_{L_{2}\left(\left[t_{0}, t\right] ; V\right)}^{2}+\alpha(h) \int_{t_{0}}^{t}\left\{\left|v^{h}(\tau)\right|_{U}^{2}-\left|u_{*}(\tau)\right|_{U}^{2}\right\} d \tau$. Here and below $\mu^{h}(\cdot)=x_{r}(\cdot)-w^{h}(\cdot)$. Setting in (1) and (4) $z=w^{h}(t)$ and $z=x_{r}(t)$, respectively, and summing the corresponding inequalities, we obtain

$$
\begin{gathered}
\left(\mu_{t}^{h}(t), \mu^{h}(t)\right)+\left\langle A \mu^{h}(t), \mu^{h}(t)\right\rangle \leq\left(B\left(u_{*}(t)-v^{h}(t)\right), \mu^{h}(t)\right)- \\
\omega^{*}\left(w^{h}\left(\tau_{i}\right)-\xi_{i}^{h}, \mu^{h}(t)\right) \quad \text { for a.a. } \quad t \in \delta_{i}=\left[\tau_{i}, \tau_{i+1}\right) .
\end{gathered}
$$

$\left(\tau_{i}=\tau_{h, i}, \xi_{i}^{h}=\xi^{h}\left(\tau_{h, i}\right)\right)$. Hence, taking into account (2) we derive a.e. in $\delta_{i}$

$$
\begin{gathered}
1 / 2 d\left|\mu^{h}(t)\right|_{H}^{2} / d t+c\left|\mu^{h}(t)\right|_{V}^{2}-\omega\left|\mu^{h}(t)\right|_{H}^{2} \leq \\
\left(B\left(u_{*}(t)-v^{h}(t)\right), \mu^{h}(t)\right)+\omega^{*}\left(w^{h}\left(\tau_{i}\right)-\xi_{i}^{h}, \mu^{h}(t)\right) .
\end{gathered}
$$

It is easy to see that for $t \in \delta_{i}$

$$
\leq\left(h+\int_{\tau_{i}}^{t}\left\{\left|w_{\tau}^{h}(\tau)\right|_{H}^{\left.\left.\left.w^{h}\left(\tau_{i}\right)-\xi_{i}^{h}, \mu^{h}(t)\right) \leq\left. x_{r \tau}(\tau)\right|_{H}\right\} d \tau\right)\left|\mu^{h}(t)\right|_{H}-\left|\mu^{h}(t)\right|_{H}^{2} .}\right.\right.
$$

In this case, for a.a. $t \in \delta_{i}$

$$
1 / 2 d\left|\mu^{h}(t)\right|_{H}^{2} / d t+c\left|\mu^{h}(t)\right|_{V}^{2} \leq\left(B\left(u_{*}(t)-v^{h}(t)\right), \xi_{i}^{h}-w^{h}\left(\tau_{i}\right)\right)+\rho_{i}(t, h, \delta),
$$

where $k_{h}=\omega^{*}+|B|(Q+d(h)), Q=\left|u_{*}(\cdot)\right|_{L_{\infty}(T ; U)}$,

$$
\rho_{i}(t, h, \delta)=k_{h}\left(h+\int_{\tau_{i}}^{t}\left\{\left|w_{\tau}^{h}(\tau)\right|_{H}+\left|x_{r \tau}(\tau)\right|_{H}\right\} d \tau\right) .
$$

Let $h_{*}>0$ such that $d(h)>Q$ for $h \in\left(0, h_{*}\right)$. Hence, using the rule of definition of a control $v^{h}(\cdot)$, we obtain

$$
\varepsilon_{h}(t) \leq \varepsilon\left(\tau_{i}\right)+2\left(t-\tau_{i}\right) \rho_{i}(t, h, \delta), \quad t \in \delta_{i} .
$$


Consequently, by Lemmas 1 and 2, the inequality

$$
\begin{gathered}
\left.\varepsilon_{h}(t) \leq \varepsilon_{h}\left(t_{0}\right)+2 k_{h}\left(h\left(\vartheta-t_{0}\right)+\delta(h)\right) \int_{t_{0}}^{t}\left\{\left|w_{\tau}^{h}(\tau)\right|_{H}+\left|x_{r \tau}(\tau)\right|_{H}\right\} d \tau\right) \leq \\
k_{1}(1+d(h))(h+\delta(h)) \quad t \in T
\end{gathered}
$$

is valid. We deduce

$$
\begin{gathered}
\Lambda^{0}\left(x_{r}(\cdot), w^{h}(\cdot)\right) \leq k_{1}(1+d(h))(h+\delta(h))+\alpha(h) k_{2}\left(1+d^{2}(h)\right), \\
\left|v^{h}(\cdot)\right|_{L_{2}(T ; U)} \leq\left|u_{*}(\cdot)\right|_{L_{2}(T ; U)}+k_{1}\left(\alpha^{-1}(h)(1+d(h))(h+\delta(h))\right)^{1 / 2} .
\end{gathered}
$$

Here, the constant $k_{1}$ does not depent on $h$ and can be written explicitly. Thus, the family $D_{h}$ is $\Lambda^{0}$-stable. It is easy to see that the condition b) of Theorem 1 holds, if

$$
y(\cdot)=x_{r}(\cdot),
$$

where $y(\cdot)=x\left(\cdot ; t_{0}, x_{0}, v(\cdot)\right)$. Prove $(21)$. Note, that

$$
\left|x_{r}(\cdot)-w^{h_{k}}(\cdot)\right|_{C(T ; H)} \rightarrow 0 \quad \text { as } \quad k \rightarrow \infty,
$$

if $h_{k} \rightarrow 0$. Here $w^{h_{k}}(\cdot)=w\left(\cdot ; t_{0}, w^{h_{k}}\left(t_{0}\right), \xi^{h_{k}}(\cdot), v^{h_{k}}(\cdot)\right), v^{h_{k}}(\cdot)=$ $D_{h_{k}}\left(\xi^{h_{k}}(\cdot)\right)$. The inequality

$$
\begin{gathered}
\left|y(t)-w^{h_{k}}(t)\right|_{H}^{2}+2 \int_{t_{0}}^{t}\left\{c\left|y(\tau)-w^{h_{k}}(\tau)\right|_{V}^{2}-\omega\left|y(\tau)-w^{h_{k}}(\tau)\right|_{H}^{2}\right\} d \tau \leq \\
2 \int_{t_{0}}^{t}\left(B\left(v(\tau)-v^{h_{k}}(\tau)\right), y(\tau)-w^{h_{k}}(\tau)\right)_{H} d \tau+ \\
\left|\omega^{*}\right| \max _{i \in\left[0: m_{h_{k}}\right]}\left|\xi_{i}^{h_{k}}-w^{h_{k}}\left(\tau_{i}\right)\right|_{H} \int_{t_{0}}^{t}\left|y(\tau)-w^{h_{k}}(\tau)\right|_{H} d \tau
\end{gathered}
$$

is true. Hence, taking into account (22), inclusion $\xi^{h_{k}}(\cdot) \in \Xi\left(x_{r}(\cdot), h_{k}\right)$, convergence $h_{k}$ to 0 , and weak convergence of controls $v^{h_{k}}(\cdot)$ to $v(\cdot)$ in $L_{2}(T ; U)$ as $k \rightarrow \infty$, in virtue of Gronwoll inequality, we obtain

$$
\left|y(\cdot)-w^{h_{k}}(\cdot)\right|_{C(T ; H)} \rightarrow 0 \quad \text { as } \quad k \rightarrow \infty .
$$

Therefore, relation (21) holds. The theorem follows from Theorem 1.

Let the following condition be fulfilled

Condition 2 The fuction $\varphi$ is differentiable and operator $C x=$ $\operatorname{grad} \varphi(x): V \rightarrow V^{*}$ is Lipshitz.

Then the estimate of convergence rate of the algorithm is true.

Theorem 3 Let $U=V, B$ be the operator of canonical embedding $V$ in $H$ and $u_{*}(\cdot)=u_{*}\left(\cdot ; x_{r}(\cdot)\right)$ be a function with bounded variation. Then the estimate

$$
\begin{gathered}
\left|u_{*}(\cdot)-v^{h}(\cdot)\right|_{L_{2}(T ; H)} \leq K\left\{\left[d(h)(h+\delta(h))+\alpha(h) d^{2}(h)\right]^{1 / 2}+\right. \\
\left.\alpha^{-1}(h) d(h)(h+\delta(h))\right\}
\end{gathered}
$$

holds.

The theorem is proved analogously to [15]. 


\section{Case $u_{*}\left(\cdot ; x_{r}(\cdot)\right) \in L_{2}(T ; U)$}

Consider the case when $u_{*}\left(\cdot ; x_{r}(\cdot)\right)$ is a norm-square integrable function, i.e., $u_{*}\left(\cdot ; x_{r}(\cdot)\right) \in L_{2}(T ; U)$. As the model $M$ we take the variational inequality (4) with the family of operators $B_{t}(\cdot ; \cdot): U \rightarrow H$ of the form (14). The family $\left(\mathcal{W}_{h}\right)$ of $t_{0}$-algorithms is defined as mentioned above, i.e., by rule (5), (11), the model control law $\mathcal{S}_{h}=\left(\Delta_{h}, \mathcal{U}_{h}\right)$ being defined by the rule (6), (7), where we assume

$$
\mathcal{U}_{h}\left(\tau_{i}, \xi_{i}^{h}, w^{h}\left(\tau_{i}\right)\right)=\arg \min \left\{l\left(\alpha, v, s_{i}\right): v \in U\right\}=-\alpha^{-1} B^{\prime} s_{i},
$$

$s_{i}=w^{h}\left(\tau_{i}\right)-\xi_{i}^{h}$. Let a family of partitions $\left(\Delta_{h}\right)$ of the interval $T$, and fuction $\alpha(h): R^{+} \rightarrow R^{+}$satisfying the following conditions:

$$
\begin{gathered}
h \delta^{-1}(h) \leq C, \quad \delta(h) \alpha^{-2}(h) \leq C, \\
\alpha(h) \rightarrow 0, \quad \delta(h) \rightarrow 0, \quad(h+\delta(h)) / \alpha(h) \rightarrow 0 \quad \text { as } \quad h \rightarrow 0+.
\end{gathered}
$$

Here $C=$ const $>0$ that does not depend on $h$.

Theorem 4 The family of positional modeling algorithms $D_{h}(8)$ of the form (4)-(7), (11), (14), (23) satisfies conditions of Theorem 1 and is regularizing.

Proof of theorem is carried out by the scheme of that for Theorem 1 . Taking into account (2) we derive a.e. in $\delta_{i}(16)$. It is also easy to see that for $t \in \delta_{i}$ inequality (17) holds along with

$$
\begin{gathered}
\left(B\left(u_{*}(t)-v^{h}(t), \mu^{h}(t)\right) \leq\left(B\left(u_{*}(t)-v^{h}(t)\right), \xi_{i}^{h}-w^{h}\left(\tau_{i}\right)\right)+\right. \\
+|B|\left\{\left|u_{*}(t)\right|+\left|v^{h}(t)\right|\right\}\left(h+\int_{\tau_{i}}^{t} g^{h}(\tau) d \tau\right),
\end{gathered}
$$

In this case, for a.a. $t \in \delta_{i}$ estimate (18) holds, provided

$$
\begin{gathered}
\rho_{i}(t, h, \delta)=\left\{\omega^{*}+|B|\left[\left|u_{*}(t)\right|_{U}+\left|v^{h}(t)\right|_{U}\right]\right\}\left(h+\int_{\tau_{i}}^{t} g^{h}(\tau) d \tau\right), \\
g^{h}(\tau)=\left|w_{\tau}^{h}(\tau)\right|_{H}+\left|x_{r \tau}(\tau)\right|_{H} .
\end{gathered}
$$

Hence, using the rule of definition of a control $v^{h}(\cdot)(23)$, we obtain

$$
\begin{gathered}
\varepsilon_{h}(t) \leq \varepsilon_{h}\left(\tau_{i}\right)+\omega^{*} \delta h+\delta \omega^{*} \int_{\tau_{i}}^{t} g^{h}(\tau) d \tau+h^{2}|B|^{2}+ \\
3 \delta \int_{\tau_{i}}^{t} F^{h}(\tau) d \tau+2 \delta^{2}\left(1+|B|^{2}\right) \int_{\tau_{i}}^{t}\left\{\left|w_{\tau}^{h}(\tau)\right|_{H}^{2}+\left|x_{r \tau}(\tau)\right|_{H}^{2}\right\} d \tau,
\end{gathered}
$$

where $F^{h}(\tau)=\left|u_{*}(\tau)\right|_{U}^{2}+\left|v^{h}(\tau)\right|_{U}^{2}$. Summing right and left hand of the inequality (25) over $i$ and taking into account Lemmas 1 and 2, the 
inclusion $u_{*}(\cdot) \in L_{2}(T ; U)$, we deduce

$$
\begin{gathered}
\varepsilon_{h}(t) \leq \varepsilon_{h}\left(t_{0}\right)+b_{1} h(1+h / \delta)+b_{2} \delta+b_{3} \delta \int_{t_{0}}^{t} F^{h}(\tau) d \tau \leq \\
\varepsilon_{h}\left(t_{0}\right)+b_{1} h(1+h / \delta)+b_{4} \delta+b_{3} \delta^{2} \sum_{j=0}^{i(t)}\left|v_{j}^{h}\right|^{2},
\end{gathered}
$$

where the symbol $i(t)$ denotes an integer part of number $t$. Besides, by the rule of definition of $v_{i}^{h}$ (see (23)), we have

$$
\left|v_{i}^{h}\right|_{U}^{2} \leq 2|B|^{2}\left(\mu_{i}^{h}+h^{2}\right) \alpha^{-2}
$$

where $\mu_{i}^{h}=\mu^{h}\left(\tau_{i}\right)=x_{r}\left(\tau_{i}\right)-w^{h}\left(\tau_{i}\right)$. From (26), (27) and inequalities $\varepsilon_{h}\left(t_{0}\right) \leq 4 h^{2}, \quad h \delta^{-1}(h) \leq C$, follows the estimation

$$
\begin{gathered}
\mu_{i}^{h} \leq \varepsilon_{h}\left(t_{0}\right)+b_{1} h(1+h / \delta)+b_{3} \delta+\alpha\left|u_{*}(\cdot)\right|_{L_{2}(T ; U)}^{2}+ \\
b_{2} \delta^{2} \sum_{j=0}^{i-1} 2|B|^{2}\left(\mu_{j}^{h}+h^{2}\right) \alpha^{-2} \leq b_{5}(h+\delta+\alpha)+b_{6} \delta^{2} \alpha^{-2} \sum_{j=0}^{i-1} \mu_{j}^{h} .
\end{gathered}
$$

Taking into account Gronwoll inequality, and inequality $\delta(h) \alpha^{-2}(h) \leq C$, we deduce

$$
\mu_{i}^{h} \leq b_{5}(h+\delta+\alpha) \exp \left\{b_{6}\left(\vartheta-t_{0}\right) \delta / \alpha^{2}\right\} \leq b_{7}(h+\delta+\alpha) .
$$

Summing left hand of the inequality (27) over $i$, we obtain from (28)

$$
\delta^{2} \sum_{j=0}^{m_{h}-1}\left|v_{j}^{h}\right|^{2} \leq 2|B|^{2} \sum_{j=0}^{m_{h}-1}\left(\mu_{i}^{h}+h^{2}\right) \alpha^{-2} \leq b_{8} \delta \alpha^{-2}(\alpha+h+\delta) .
$$

Due to (24) without commonness loss, suppose $\delta(h) \alpha^{-1}(h) \leq 1$. Therefore, from (26), (29) we derive the estimation

$$
\varepsilon_{h}(t) \leq b_{7}\left(h+\delta+\delta^{2} \alpha^{-2}+h \delta \alpha^{-2}\right) \leq b_{8}(h+\delta) .
$$

Therefore $\left|v^{h}(\cdot)\right|_{L_{2}(T ; U)}^{2} \leq\left|u_{*}(\cdot)\right|_{L_{2}(T ; U)}^{2}+b_{8}(h+\delta) \alpha^{-1}$. This means that the inequality $(9)$ takes place if we have $k_{1}(h)=1, k_{2}(h)=\left(b_{8}(h+\right.$ $\left.\delta(h)) \alpha^{-1}(h)\right)^{1 / 2}$. From $(30)$ we deduce $(10)$, where functional $\Lambda^{0}(\cdot, \cdot)$ is defined by $(15)$ and $k_{3}(h)=b_{9}(h+\delta(h)+\alpha(h))$. The theorem is proved.

The next theorem is proved analogously to Theorem 3:

Theorem 5 Let conditions of Theorem 3 hold. Then the estimate

$$
\left|u_{*}(\cdot)-v^{h}(\cdot)\right|_{L_{2}(T ; H)} \leq K\left\{(h+\delta(h))^{1 / 2}+(h+\delta(h)) \alpha^{-1}(h)\right\}
$$

is true. 


\section{References}

[1] Brezis, H. (1973). Operateurs maximaux monotones et semigroupes de constractions dans les espaces de Hilbert. Amsterdam-London-New York.

[2] Barbu, V. (1984). Optimal control of variational inequalities. Pitman Anvanced Publishing Program, London.

[3] Duvaut, G. and Lions, J.-L. (1972). Les inéquations en mécanique et en physique. Dunod, Paris.

[4] Glowinski, R., Lions, J.-L. and Trémolières, R. (1980). Numerical analysis of variational inequalities. North-Holland, Amsterdam.

[5] Kryazhimskii, A. V. and Osipov, Yu. S. (1983). Modelling of a control in a dynamic system. Engineering Cybernetics, 21:38-47. in Russian.

[6] Kryazhimskii, A. V., Maksimov, V. I. and Osipov, Yu. S. (1983). On positional simulation in dynamic systems. J. Appl. Math. Mech., 47:709-714.

[7] Osipov, Yu. S., Kryazhimskii, A. V. and Maksimov, V. I. (1991). Dynamic regularization problems for distributed parameter systems. Sverdlovsk, Russia. in Russian.

[8] Kryazhimskii, A. V. and Osipov, Yu. S. (1995). Inverse problems for ordinary differential equations: dynamical solutions. Gordon and Breach, London.

[9] Maksimov, V. I. (1988). Dinamical modeling of unknown disturbances in parabolic variational inequalities. Prikl. Mat. Mech., 52:743-750. in Russian.

[10] Maksimov, V. I. (1992). Inverse problems for variational inequalities. Internat. ser. of Numer. Math., 107:275-286. Birkhäuser Verlag, Basel.

[11] Maksimov, V. I. (1990). Stable reconstruction of unknown disturbances in parabolic variational inequalities. In: Problems of Optimization and stability in Control Systems. UB AS USSR, Sverdlovsk. 74-86. in Russian.

[12] Pandolfi, L. and Maksimov, V.I. (2000). Dynamical reconstruction of unbounded controls in nonlinear dynamical systems. Proceedings CD of the Fourteenth International Symposium of Mathematical Theory of Networks and Systems MTNS , Perpignan, France, June 19-23, 2000.

[13] Maksimov, V. (1998). Dynamical inverse problems for parabolic variational inequalities. An. St. Univ. Ovidius Constanta, 6:97-110.

[14] Osipov, Yu. S., Kryazhimskii, A. V. and Maksimov, V. I. (2000). Dynamical inverse problems for parabolic systems. Differential Equations, 36:579-597. in Russian.

[15] Maksimov, V. I. (2000). The problems of dynamical reconstruction of inputs of infinite-dimensional systems. Ekaterinburg. in Russian.

[16] Kappel, F., Pandolfi, L., Maksimov, V. and Blizorukova, M. (2000). Problems of dynamical identification in nonlinear differential systems. Proceedings of the Polish-German symposium on "Science Research Education" (SRE'2000), 5156.

[17] Krasovskii, N. N. and Subbotin, A. I. (1988). Game-theoretical control problems. Springer. 